\title{
And now, Weyl fermions
}

Topological semimetal and Fermi-arc surface states in the electronic structure of pyrochlore iridates,

X. Wan, A.M. Turner, A. Vishwanath and S.Y. Savrasov, Phys. Rev. B 83, 205101(2011), arXiv:1007.0016

Recommended with a commentary by Vivek Aji, University of California Riverside.

With Majorana and Dirac fermions already part of the condensed matter vocabulary, it didn't take long before Weyl fermions made their appearance.

Sublattice symmetry and time reversal invariance in graphene results in the conduction and valence band touching at two distinct points in the Brillouin zone. The dispersion in the vicinity of these point is linear. Each band is doubly degenerate (spin) and thus there are

four states at the nodes. It was soon realized in such a model strong spin-orbit scattering will open up a gap, which leads to a topologically nontrivial insulating state. Such a state has protected edge modes, reminiscent of the physics of the Quantum Hall Effect. The topological aspect has led to the discovery of the topological insulator: materials that are Bloch insulators in the bulk with protected metallic edge and surface states in two and three dimensions respectively. Unlike graphene, the two bands that touch at the surface of the topological insulator are singly degenerate. Both these examples, graphene and topological insulators, involve two dimensional systems described by a two component Dirac equation. Is it possible to realize a three dimensional analog which would be the Weyl fermions ?

One year after Dirac's theory of the electron appeared in 1928, Weyl showed that a two component version is sufficient to describe massless fermions. The conservation of chirality breaks the four component description into two sectors that do not mix. Weyl observed that each sector alone describes a particle with no associated antiparticle. The theory violates parity, which prompted Pauli to reject the notion of massless fermions. Unfortunately Weyl did not live long enough to celebrate the discovery of Neutrinos and witness the resurgence of interest in his work.

To have Weyl fermions in solid state systems, one requires two and only two states to be degenerate at a finite number of points in the Brillouin zone. This needs fine tuning as the diagonal elements of the $2 \times 2$ Hamiltonian should be equal and the off diagonal elements 
zero. In addition symmetry considerations also prove quite stringent. The Hamiltonian at a given node is $\pm v \vec{\sigma} \cdot \vec{p}$, where the $\operatorname{sign}$ determines the Helicity, $\vec{\sigma}=\left\{\sigma_{x}, \sigma_{y}, \sigma_{z}\right\}$ is a vector of Pauli matrices and $\vec{p}$ is the momentum. Under inversion $\vec{p} \rightarrow-\vec{p}$. If inversion is preserved, a Weyl node at $\vec{p}$ is always accompanied by a Weyl node at $-\vec{p}$ with opposite chirality. On the other hand if time reversal is preserved than there is also a node at $-\vec{p}$ with the same chirality, as both $\vec{p}$ and $\vec{\sigma}$ change sign. This implies that if both symmetries are preserved every Weyl node, if it exists, is a doublet of nodes with opposite chiralities. Such doublets are nothing but the four component Dirac fermions and are susceptible to opening up of gaps. Thus, to get stable Weyl nodes, one or the other symmetry needs to be broken.

What Wan et al. find is that the pyrochlore iridates have the possibility of hosting Weyl semi-metallic phase, when the magnetic state is such that time reversal is broken but inversion is preserved. Irridates have strong spin-orbit coupling, a characteristics favorable in stabilizing topological insulators. In addition the Coulomb interaction, modeled by an on site Hubbard $\mathrm{U}$, is moderate enough to allow for itinerancy to compete with Mott insulating tendencies. Using an $\mathrm{LDA}+\mathrm{SO}+\mathrm{U}$ approach, they explore the nature of the ground state as a function of $\mathrm{U}$.

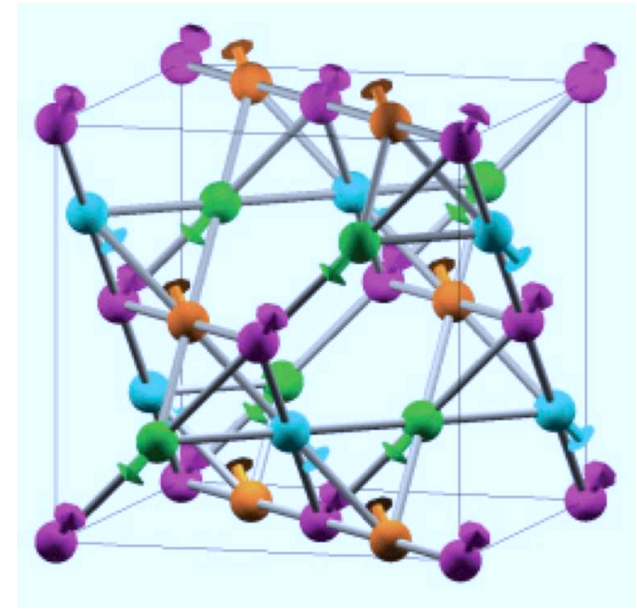

FIG. 1: The $4 \mathrm{in} / 4$ out magnetic pattern is shown. Inversion is preserved and the a Weyl semi metallic state is realized.

For intermediate $U$ they find the most favorable magnetic structure is one with the spins on the tetrahedra arranged in a 4 in/ 4 out pattern (see fig.1).The low energy behavior is that of 24 Weyl nodes (see fig.2), three each around the eight $L$ points of the Brillouin zone. 
The two bands that touch have total angular momentum $J=1 / 2$ with opposite parity. These crossings are protected by inversion symmetry of both the crystal and the magnetic order. While accidental degeneracies are realizable in nature, what makes this system unique is that the nodes are at the Fermi surface and control the low energy physics.

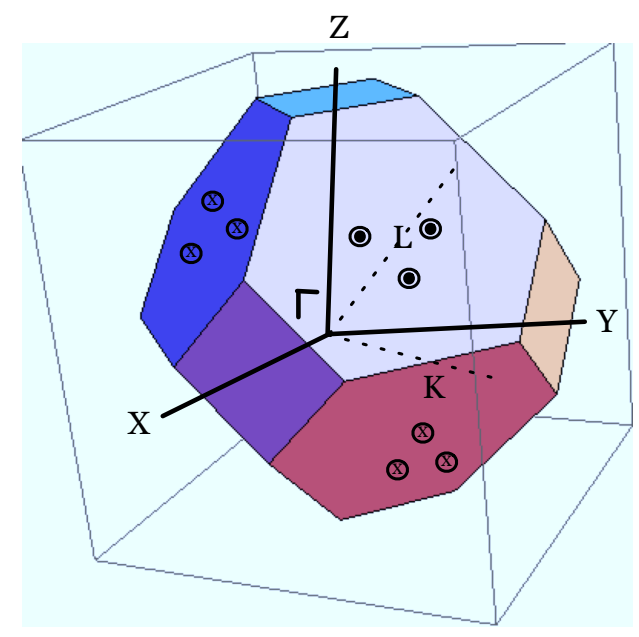

FIG. 2: There are 24 Weyl nodes located in the vicinity of the L points. Nodes with opposite felicities are marked by a dot and a cross respectively.

Weyl semi-metals also host protected surface states. Unlike quantum hall or the topological insulator, the bulk is gapless and yet these surface states are robust. In momentum space they lead to Fermi arc that connect two bulk Weyl nodes of opposite chirality. Fermi surfaces are usually closed and separate occupied and unoccupied states in the Brillouin zone. The termination of a Fermi surface in the Weyl semimetal is facilitated by the intersection with the bulk bands. Establishing the existence of and exploring the consequence of such Fermi arcs is one of the most intriguing direction of research to pursue.

The magneto-electric properties of such semi-metals are also unconventional. In a magnetic field, the zeroeth Landau level disperses linearly, with the sign of the velocity determined by the helicity of the node. In the presence of an electric field, particles are transferred from below the fermi energy to above at one node, while the reverse happens at the node with opposite chirality. In the context of relativistic field theories, this phenomena gives rise to the Adler-Bell-Jackiw anomaly (i.e. spontaneous generation of fermonic charge). In the condensed matter context, the same physics results in an anomalous magneto-conductivity. The key difference in the latter is that the vacuum is the occupied fermi sea with a well 
defined bottom of the band. One expects a transfer of particles between nodes of opposite chirality as opposed to the generation of charge from a Dirac vacuum with infinite supply of particles.

The field of Weyl semi-metals is in its infancy, but searches for possible realization are being explored both experimentally and theoretically. For example, layered heterostructures of normal and magnetically doped 3D topological insulators have been shown to stabilize a Weyl semimetal [1]. More generally the establishment of a whole new class of material phenomena linked to underlying topological nature makes this an exciting time in condensed matter physics. I am sure there is more to come.

[1] Weyl Semimetal in a Topological Insulator Multilayer, A.A. Burkov and L. Balents Phys. Rev. Lett. 107, 127205 (2011). 